\title{
Internet Protocol Function Enhancement using Information Centric Approach to Solve Mobility and Security Problems for Internets of Things
}

\author{
Azana Hafizah Mohd Aman ${ }^{1}$, Rosilah Hassan² \\ \{azana@ukm.edu.my¹, rosilah@ukm.edu.my² \} \\ FTSM, Universiti Kebangsaan Malaysia, Bangi 43600, Malaysia. ${ }^{12}$
}

\begin{abstract}
In order to facilitate automated tasks, technologies embrace smart devices and smart applications of Internet of Things (IoT) environment. The IoT environment is blended with various contexts and services that require high quality performance in terms of mobility and security. It is challenging for IoT environment to have seamless mobility with secure and reliable communication due to its nature constraint oriented characteristic such as low processor, limited memory and low energy. The challenges are worsen when the current Internet Protocol networking does not cater mobility and security problem of IoT constraint oriented devices because of its host cache identity, large packet size and time consuming complex multi-layer functions. These challenges have motivated us to enhance the Internet Protocol networking function to overcome existing mobility and security limitations for IoT environment using Information Centric networking approach. The main reason for choosing Information Centric networking is because of the connection is based on data instead of machine and addresses, it is data security oriented and it also provides in-network caching that are useful for constraint oriented communication.
\end{abstract}

Keywords : Information Centric , IoT mobility, IoT security, Internet of Things

\section{Introduction}

IoT environment consist of blended technologies and services that integrate data from various contexts. IoT environment has many challenges such as mobility, security, scalability, and communication reliability [1],[2],[3]. IoT devices are mostly considered as lossy with low bandwidth network consumption due to capacity constraint of processor, memory and energy. Therefore IoT environment only use small packet byte size for communication. This resulted in fragmentation of large packet byte size key exchange message required by existing security binding of Internet Protocol networking function. Hence, allowing Denial of Service (DoS) attack, an attack vector for state exhaustion. This also leads to lower system performance due to retransmission and fragment loss. Internet Protocol networking function with many layered approaches and protocols bonded together worsen the performance for IoT environment [3]. Some of these layered protocol required negotiation that contribute to increase number of exchange signalling messages and computation overhead [3], [4]. These make data mobility impossible to be available in timely manner, everywhere with reliable connectivity for IoT 
environment. Figure 1 highlighted the mobility and security of data from the Malaysia National IoT Strategic Roadmap: IoT components perspective (Figure 1).

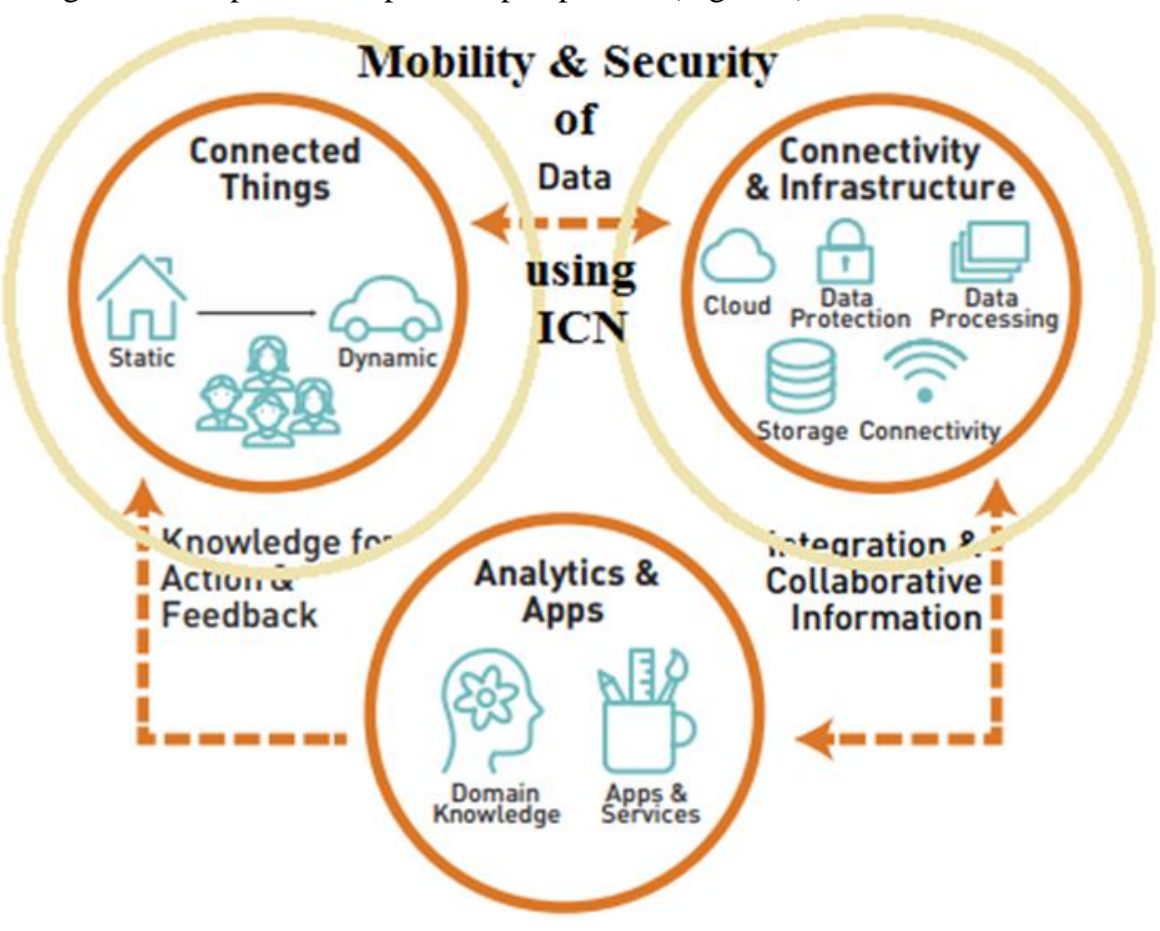

Fig. 1 Mobility and Security of Data from the IoT Strategic Roadmap Perspective.

Hence it is crucial to make research in the following aspects:

1. Study the effect of using in network caching, data connection oriented and data security oriented of the Information Centric Networking to solve IoT Internet Protocol networking mobility and security problems.

2. Enhance the IoT Internet Protocol networking using Information Centric networking approach.

3. Investigate the controlling parameter values in Information Centric approach in order to comply with lossy and capacity constrained IoT devices.

4. Combine the three components: network caching, data connection oriented and data security oriented in order to enhance the performance of the proposed Information Centric approach.

The hypothesis of this idea is, if in-network caching, data connection oriented and data security oriented of Information Centric networking approach can be combined intelligently, then employing these criteria will enhance the performance of IoT communication and solve the mobility and security problems. In order to test the hypothesis, the following research questions are formulated and Fig 2 summarized the research ideas (Figure 2). 
1. How to use in-network caching, data connection oriented and data security oriented to solve IoT mobility and security problems?

2. How to effectively enhance the Internet Protocol networking messages flow and format using Information Centric networking approach?

3. How to enhance the networking parameter control: cache size and location, types of data naming, data authentication?

4. How to combine the three improvement components: in network caching, data connection oriented and data security oriented to enhance the performance of the proposed networking?

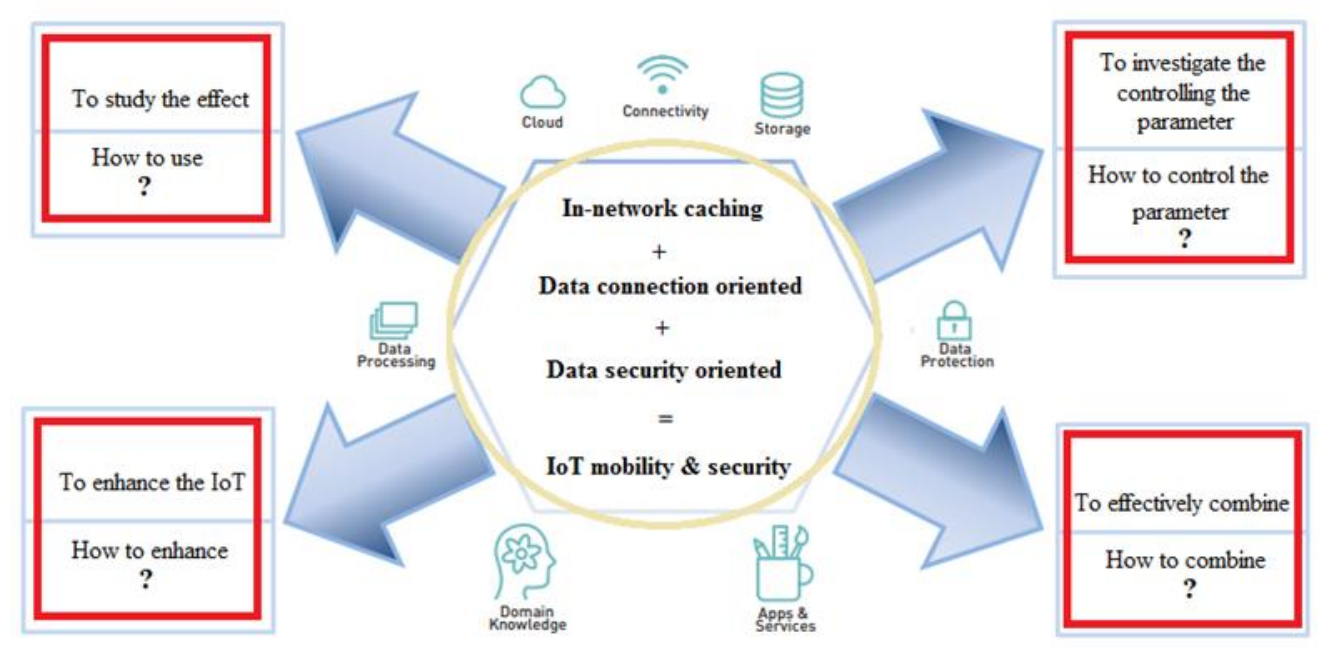

Fig. 2. Summary of the research ideas.

This paper is structured as follows: section 2 delivers research background. Section 3 describes the research methodology. Followed by the conclusion in section 4 .

\section{Research background}

Current challenges of IoT using Internet Protocol networking are discussed in [4]. Among IoT Internet Protocol networking problems are mobility, security, energy management, scalability, connection interoperability, big data, and identification. Patches are

implemented on Internet Protocol networking to enable IoT communication. This efforts added layers and gateways to cater IoT environment, causing too much configuration with complex protocol stack, large packet size and without security consideration. Mobility involve device discovery, it is the establishment of relationships among nodes. In the actual IoT Internet Protocol networking, the relationship service requires the mapping of Internet Protocol addresses to the physical node. This mapping involves configurations which are 
not efficient in the dynamic IoT environment. In mobility, network performance is very critical as a bad network means service interruption, data loss, and serious impact on the application functionality due to physical movement. This work will focus in solving mobility and security problems of IoT environment using Information Centric networking approach.

Information Centric networking is proposed as future communication concept and more effective IoT environment support [4],[5],[6]. Information centric networking is based on data connection oriented, instead of machine or devices addresses and based on data security oriented, a direct secure of data objects. Information Centric networking transforms the current connection concept of resources sharing to information sharing and transforms the current communication model from Host-centric to Information-centric. Hence it is highly capable to support low-level, constraint capacity devices for higher level services and application level goals [7]. It is less resource-consuming and more lightweight, the innetwork caching and forwarding indicate higher reliability and lower sensitivity to disruptions than end-to-end Internet Protocol solutions [7]. In order to validate the hypothesis, qualitative and

quantitative analysis has to be done.

Many researchers use network simulators methods to evaluate Internet Protocol networking problems [8]; some of current network simulators are NS-3 [9], OMNeT [10], OPNET [11], GNS3 [12] and QualNet [13]. Few researchers considered mathematical evaluation [14], [15] which is able to search for optimal small size topology or environment but very time consuming for larger size topology. Hence we will use both mathematical and network simulators methods to evaluate Internet Protocol networking and Information centric networking with the appropriate values that will be used for the evolutionary parameters, making it more efficient to overcome IoT mobility and security problems.

This research idea is relevance to Malaysia Government Policy, is aligned with the following policies (Figures 3 and 4 ) :

1. National Policy on Industry 4.0 [16] - Point out a goal which is in line with the United Nation's Sustainable Development Goals (SDGs) goal number 9: to provides technological solutions for a better industrialisation as shown in Figure 3 and Figure 4.

2. National Cyber Security Policy [17] - Thrust number 5 which is research and development towards self-reliance

3. National IoT Strategic Roadmap [18] - Goal number 3 which is to create a conducive IoT industry ecosystem 


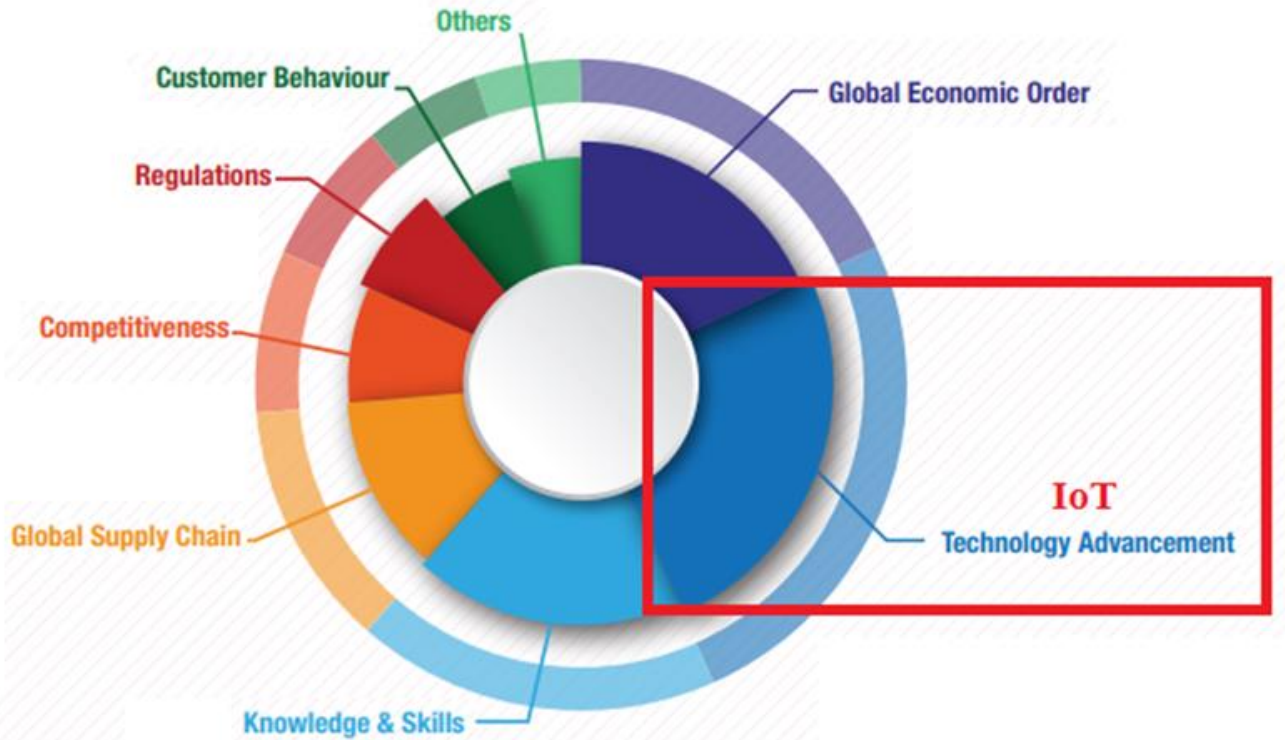

Fig. 3 ICN from Malaysia Industry 4.0 Perspectives.

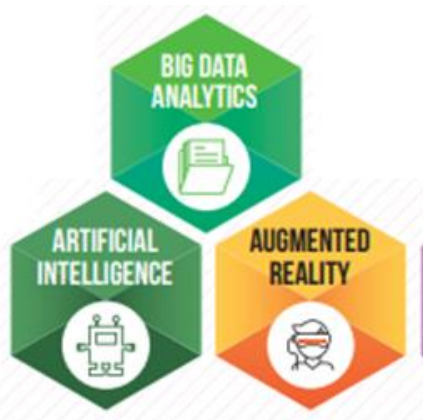

IoT

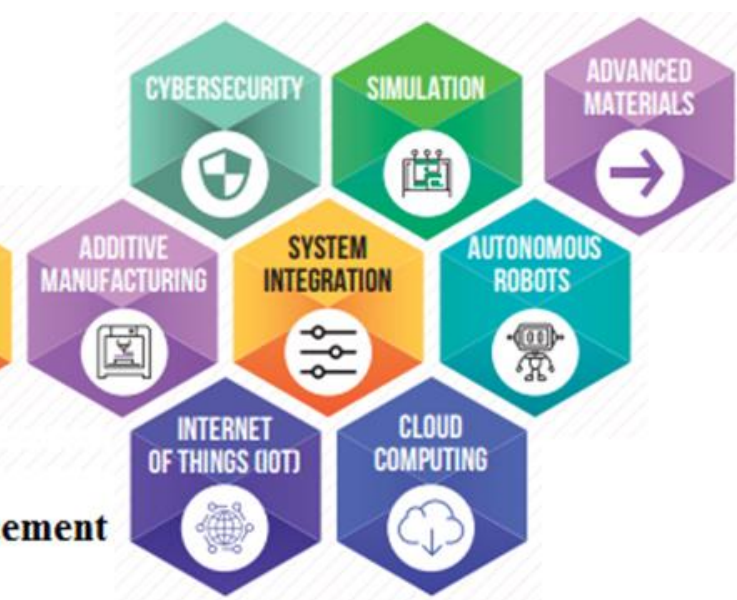

Fig 4. ICN potential applications from Malaysia Industry 4.0 technologies perspectives.

\section{Research Methodology}

This research idea consist of three main stages as summarized in Fig 5, elaborated in Table 1 and while the research activities flow chart is summarized in Fig 6: 
Table 1. Research Idea Stages.

\begin{tabular}{|c|c|}
\hline Stages & Description \\
\hline Stage 1 & $\begin{array}{l}\text { Study and investigate below criteria: } \\
\text { 1) IoT los sy and capacity constrained environment mobility and security requirem } \\
\text { in terms of response time, battery capacity, memory size, packet size and othercri } \\
\text { information. } \\
\text { 2) In network caching, data connection oriented and data security orientec } \\
\text { information centric networking in terms of communication process flow, sign: } \\
\text { packet size, number of signaling messages, cache size, cache availability, c: } \\
\text { location, data authentication type and other critical information. } \\
\text { 3) Publisher and requestor of information centric networking packet format and c } \\
\text { packet information requirement } \\
\text { 4) Existing Internet Protocol networking infrastructure for IoT in terms of process } \\
\text { and packet format }\end{array}$ \\
\hline Stage 2 & $\begin{array}{l}\text { Framework design } \\
\text { This phase focuses on how to initialize solution and satisfy the mobility and secl } \\
\text { requirement of IoT environment. In order to accomplish this goal, by using sta } \\
\text { results the baseline topology for the proposed IoT Information Centric networking } \\
\text { be logically drawn. Based on the baseline topology design, the pseudo code/flowc } \\
\text { communication process, mathematical models and network simulators algorithm } \\
\text { then be developed. The IoT Information Centric topology baseline and the algorj } \\
\text { will then be scalable by varying the number of publisher/requestor, publisher/requi } \\
\text { speed, network area size, in network cache size and publisher/requestor authentic } \\
\text { type. }\end{array}$ \\
\hline Stage 3 & $\begin{array}{l}\text { Evaluation: algorithm verification and results analysis } \\
\text { The proposed approach will be analyzed using mathematical and network simula } \\
\text { algorithm. The mathematical part will be done using MATLAB or advance MS O } \\
\text { Excel functions. While for the network simulators algorithm part, a Virtual Mac } \\
\text { (VMWARE workstation Pro } 5 \text { ) with network simulators (GNS3, NS3 and OMNeI } \\
\text { will be used to run the algorithm and traffic tracer software (Wireshark). The net } \\
\text { performance will be evaluated and benchmarked with existing Internet Prot } \\
\text { networking based on general metrics such as service recovery time, signaling } \\
\text { packet loss cost and othercritical metrics. }\end{array}$ \\
\hline
\end{tabular}

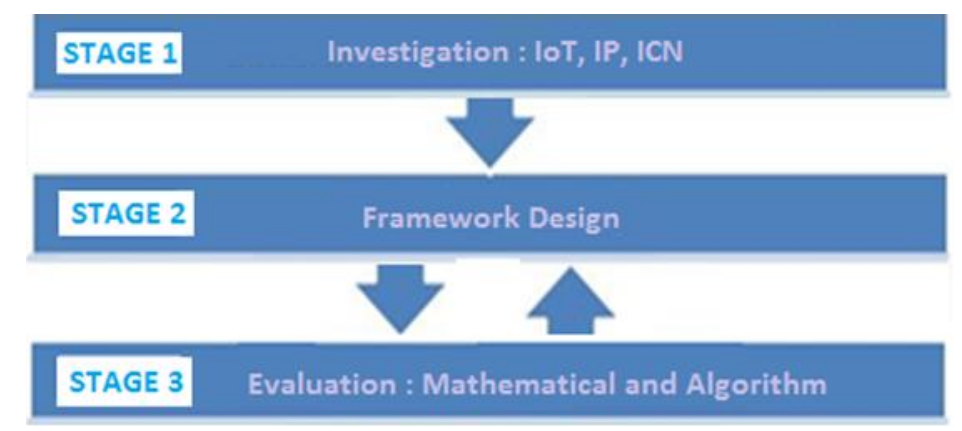

Fig. 5. Research Stages. 


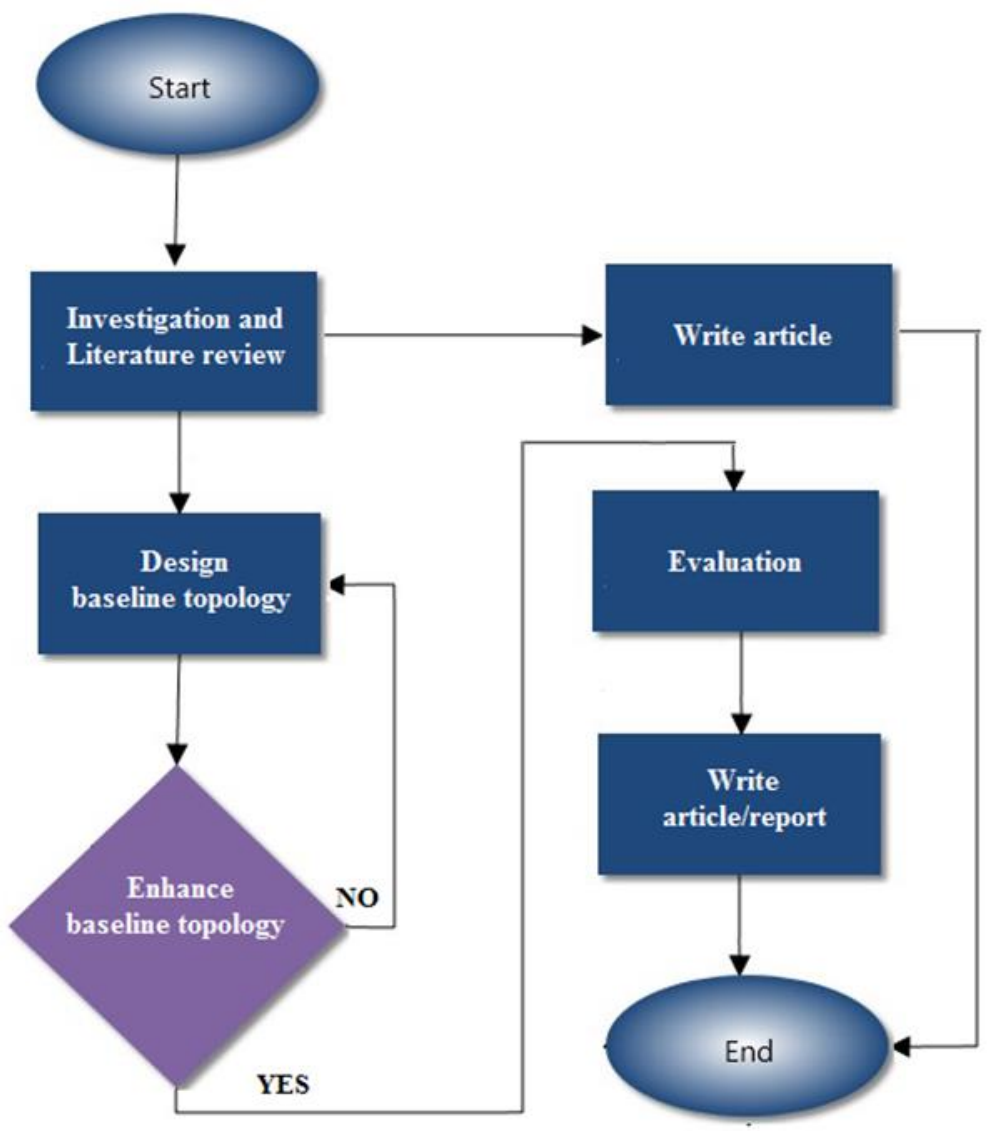

Fig. 6. Research Activities. 
Table 2 described the relationship between objectives, stages and questions.

Table 2. Objectives, Stages and Questions.

\begin{tabular}{|c|c|c|}
\hline Objectives & S tages & Questions \\
\hline $\begin{array}{l}\text { 1. To study the effect of using in network } \\
\text { caching, data connection oriented and data } \\
\text { security oriented of the Information Centric } \\
\text { Networking to solve IoT Internet Protocol } \\
\text { networking mobility and security problems. }\end{array}$ & Stage 1 & $\begin{array}{l}\text { 1. How to use in-network cach } \\
\text { connection oriented and data } \\
\text { oriented to solve IoT mobility an } \\
\text { problems? }\end{array}$ \\
\hline $\begin{array}{l}\text { 2. To enhance the IoT Internet Protocol } \\
\text { networking using Information Centric } \\
\text { networking approach. }\end{array}$ & Stage 2 & $\begin{array}{l}\text { 2. How to effectively enhance th } \\
\text { Protocol networking messages } \\
\text { format using Information } \\
\text { networking approach? }\end{array}$ \\
\hline $\begin{array}{l}\text { 3. To investigate the effect of controlling the } \\
\text { parameter values in the proposed } \\
\text { Information Centric approach in order to } \\
\text { comply with lossy and capacity constrained } \\
\text { IoT devices. }\end{array}$ & Stage 3 & $\begin{array}{l}\text { 3. How to enhance the } \\
\text { networking parameter control: c } \\
\text { and location, types of data nan } \\
\text { authentication? }\end{array}$ \\
\hline $\begin{array}{l}\text { 4. To effectively combine the three } \\
\text { components: network caching, data } \\
\text { connection oriented and data security } \\
\text { oriented in order to enhance the performance } \\
\text { of the proposed Information Centric } \\
\text { approach. }\end{array}$ & Stage 3 & $\begin{array}{l}\text { 4. How to combine the three imp } \\
\text { components: in network cachi } \\
\text { connection oriented and data } \\
\text { oriented to enhance the performal } \\
\text { proposed networking? }\end{array}$ \\
\hline
\end{tabular}

\section{Conclusion}

The idea of this paper is to proposed research to enhance Internet Protocol networking function using Information Centric networking approach for seamless mobility and secure connections of IoT environment. The enhanced networking approach has the potential to be used in IoT communication especially for lossy and low capacity IoT devices network that are normally used in smart vehicles, smart homes, smart hospitals and smart industries. The output of this research contributes a significant impact for the IoT technology. IoT networking that support seamless mobility and security for lossy constrained devices can ensure high communication performance especially for smart technologies in vehicles, homes, and industries. This research will help our Malaysia to achieve the implementation of national IoT strategic roadmap, national cyber security policy and national industrial 4.0 policy.

\section{References}

[1] Triantafyllou, A. Sarigiannidis, P. Lagkas, T. D.: Network Protocols, Schemes, and Mechanisms for Internet of Things (IoT): Features, Open Challenges, and Trends. WILEY Hindawi Wireless Communications and Mobile Computing. Vol. 2018 (2018)

[2] Garcia-Morchon, O. Kumar, S. Sethi, M.: State-of-the-Art and Challenges for the Internet of Things Security, IETF Work in Progress (2018)

[3] Shang, W. Yu, Y. Droms, R. and Zhang, L.: Challenges in IoT Networking via TCP/IP Architecture. (2016) 
[4] Arshad, S. Azam, M. A. Rehmani, M. H. Loo, J.: Recent Advances in Information-Centric Networking based Internet of Things (ICN-IoT). IEEE Internet Of Things Journal. Vol. 14, No. 8, (2018)

[5] Ravindran, R. Ed, Zhang, Y. Grieco, L. Lindgren, A. Raychadhuri, D. Baccelli, E. Burke, J. Wang, G. Ahlgren, B. Schelen, O. : Design Considerations for Applying ICN to IoT. IRTF ICNRG. Work in Progress (2018) .

[6] Shang, W. Bannis, A. Liang, T. Wang, Z. Yu, Y. Afanasyev, A. Thompson, J. Burke, J. Zhang, B. and Zhang, L. : Named Data Networking of Things. IEEE Explore (2016)

[7] Gundogan, C. Schmidt, T. and Waehlisch, M. : Publish-Subscribe Deployment Option for NDN in the Constrained Internet of Things. ICN Research Group (2018)

[8] Azana Hafizah Mohd Aman, Aisha-Hassan Abdalla Hashim, Abdullah, A. and Ramli, H. A. M. : Network Simulators Parametric Comparison for Network Mobility Management. International Journal of Future Generation Communication and Networking. Vol.9 (10), pp. 1-11 (2016)

[9] Network Simulator NS-3,

[10] OMNeT++ Discrete Event Simulator,

[11] OPNET Modeler or Riverbed Modeler,

[12] GNS3, https://www.gns3.com

[13] QualNET, https://www.scalable-networks.com/qualnet-network-simulator-software

[14] Azana Hafizah Mohd Aman, Aisha-Hassan Abdalla Hashim, Abdullah, A. and Ramli, H. A. M. : Advance Signaling Cost for Multicast Fast Reroute Proxy Mobility Management. Indian Journal of Science and Technology, Vol. 9 (25), (2016)

[15] Azana Hafizah Mohd Aman, Aisha-Hassan Abdalla Hashim, Abdullah, A. and Ramli, H. A. M. : Evaluation of an Enhanced Multicast Data Flow Technique in Network Mobility. International Journal of Future Generation Communication and Networking, Vol.9 (7), pp.153-164 (2016)

[16] Malaysia National Policy on Industry 4.0, MITI,

[17] Malaysia National Cyber Security Policy, MOSTI,

[18] Malaysia National Internet of Things (IoT) Strategic Roadmap, MIMOS Berhad, mimos.my/iot/National_IoT_Strategic_Roadmap_Book.pdf. 